\title{
Nanoscale Properties of Biopolymer Multilayers
}

\author{
Z.A. Gezgin ${ }^{\mathrm{a}, \mathrm{b}^{*}}$, T.C. Lee ${ }^{\mathrm{a}}$ and Q. Huang ${ }^{\mathrm{a}}$ \\ a Department of Food Science, Rutgers University, 65 Dudley Road, New Brunswick, New \\ Jersey 08901-8520, USA \\ ${ }^{\mathrm{b}}$ TUBITAK, The Scientific and Technological Research Council of Turkey, Tunus Caddesi \\ No: 80, 06100, Kavaklıdere/Ankara, TURKEY
}

* Corresponding author Tel: +905301081617; Fax +1732-932-6776; E-mail zaferaligezgin@hotmail.com 


\begin{abstract}
Layer by Layer (LbL) deposition is a simple and inexpensive method of multilayer self-assembly, that relies on the electrostatic interactions of oppositely charged polyelectrolytes. Functional biopolymers used to build such nanostructured multilayers can keep serving their inherent functions in food systems. A good example is chitosan, a positively charged biopolymer which has antimicrobial properties and forms stable complexes with negatively charged polymers. This study investigates the potential of commercially available food grade biopolymers to substitute synthetic polyelectrolytes when building nanostructured multilayers. Chitosan molecular weight, carrageenan charge density and pectin degree of esterification and amidation was varied to investigate their respective effects on the nanoscale morphology and wettability of these surfaces. Extracellular Ice Nucleators (ECINs), functional lipoglycoproteins of negative charge known to increase ice nucleation temperatures in food systems, were used to confirm the suitability of biopolymer multilayers for fabricating functional food grade nano-thin films. High molecular weight chitosan and iota-carrageenan created the most appropriate biopolymer pair to serve this purpose, as demonstrated by the nanoscale evaluation of these ultra-thin layers. ECINs successfully adsorbed onto the multilayer system, and ice nucleation activity of the nano-thin film was confirmed via the cooling bath experiments.
\end{abstract}

\title{
Keywords
}

Layer by Layer Deposition (LbL), chitosan, pectin, carrageenan, Extracellular ice nucleators (ECIN), atomic force microscopy (AFM), surface wettability, water contact angles. 


\section{Introduction}

Layer by Layer (LbL) deposition technique is an alternative way to fabricate thin films on surfaces to the traditional Langmuir-Blodgett method which requires special instruments and presents limitations to the substrate size and shape, as well as film stability and quality (Decher, 1997). LbL deposition relies mainly on the electrostatic interactions of oppositely charged polyelectrolytes for multilayer self-assembly (Zhang, Chen \& Zhang, 2007). This allows the building of multilayer systems more stable than those obtained by physical adsorption, due to the stronger electrostatic attraction among the layers and with the substrate (Cai, Rechtenbach, Hao, Bossert \& Jandt, 2005). There are only two basic requirements for LbL deposition; one is that the polyelectrolytes of interest are soluble in water and the other that they carry opposite electrostatic charges. The LbL technique has several advantages such as (1) it is very easy to perform and no expensive or complicated instruments are required, (2) different building blocks can be incorporated into the multilayer system to obtain the desired properties and (3) it can be applied to any charged substrate, of any shape or size (Zhang et al., 2007).

Nano-thin multilayers nanostructured from oppositely charged proteins or polysaccharides provide perfect foundations for delivering functional substance making up the top layer of the multilayer system, such as negatively charged Extracellular Ice Nucleators (ECINs). ECINs are substances that can initiate nucleation by organizing water molecules into an ice-like pattern at relatively high temperatures $\left(-2^{\circ} \mathrm{C}\right)$ (Duman, 2001). They are used in food systems to increase freeze-thaw resistance, modify food texture and deliver energy savings in freeze drying and concentration applications (Li-Jung, Chen, Tzeng, Chiou \& Jiang, 2005; Zhu \& Lee, 2007) There are no reports regarding the activity of ice nucleators when applied as a nano-thin layers in food products. 
ECIN nano-thin films have been fabricated using non-food grade synthetic oppositely charged cationic poly (diallyldimethylammonium chloride) and anionic poly (styrenesulfonate) (Gezgin, Lee \& Huang, 2013). Thus it is of importance to consider the fabrication of ECIN nano-thin films using food grade polyelectrolytes.

Chitosan is a positively charged biopolymer carrying amine groups in its structure. It is obtained by the deacetylation of chitin (an exoskeleton structure-forming polysaccharide obtained during krill, shrimp, and crab processing as a by-product) under alkaline conditions and it is the second most abundant renewable organic resource after cellulose (Shumilina \& Shchipunov, 2002). At a deacetylation degree of $85 \%$, chitosan holds one ammonium group for approximately each 1.2 saccharide unit, which allows it to form complexes with negatively charged polymers (Kujawa, Moraille, Sanchez, Badia \& Winnik, 2005).

Carrageenan is a generic name for high sulfate containing, linear, anionic heteropolysaccharide, commercially extracted from red marine algae (Bartkowiak \& Hunkeler, 2001). This family of sulfated galactans are categorized by their charge densities; as lambda, iota, kappa carrageenans and furcelleran (aka Danish Agar, a polysaccharide very similar to kappa carrageenans in structure) having 2.07, 1.53, 0.92 and 0.69 sulfate groups per disaccharide unit, respectively (Hugerth \& Sundelöf, 2001).

Pectins are polysaccharides made up of D-galacturonic acid units connected via the $\alpha$ (1-4) linkages. Carboxyl groups are commonly esterified as methoxyl groups in nature, and the percentage of esterified groups, a.k.a. degree of esterification, is an important parameter in their classification as either high methoxyl (HM, >50\%) or low methoxyl (LM, < 50\%) pectins (Worth, 1967). Thus, the aims of this work were to: (a) investigate the potential of chitosan, pectin and carrageenan ability to form food grade multilayer systems via the LbL 
deposition technique, with ECINs adsorbed onto the multilayer system; and (b) evaluate the ice nucleation activity of the nano-thin film via the cooling bath experiments.

\section{Materials and methods}

\subsection{Materials}

Chitosans of $1 \mathrm{kDa}$ and $10 \mathrm{kDa}$ molecular weight were purchased from Kitto Life Co. Ltd. (Seoul, Korea) and 44, 130 and 330 kDa from Kunpoong Bio. Co. Ltd. (South Korea). Degree of deacetylation (DOD) for all chitosans was in the range of 98-99\%. Pectins with different degrees of esterification (DE) and amidation (DA) were acquired from Danisco (Grindsted, Denmark) and Sigma Aldrich (St. Louis, MO) (Danisco Grindsted LA 410-DE:2933\%,DA:20\%, LC 950-DE:31-33\% and Sigma P9561-DE:90\%). Furcellaran was a gift from

FMC Biopolymer (Princeton, NJ). Kappa carrageenan (type I, CAS\# 9000-07-1), iota carrageenan (type II, CAS\# 9062-07-1), lambda carrageenan (type IV, 9064-57-7) were purchased from Sigma Aldrich (St. Louis, MO). ECINs produced by Erwinia herbicola subsp. ananas, (Cat. No. 11530, ATCC, Rockville, MD) was kindly provided by Prof. T.C. Lee's group. $\mathrm{H}_{2} \mathrm{O}_{2}$ and $\mathrm{H}_{2} \mathrm{SO}_{4}$ were purchased from Sigma-Aldrich (Milwaukee, WI), and all chemicals were used as received without further purification. Silicon wafers were purchased from Montco Silicon Technologies (Spring City, PA). Ultrapure water (Milli-Q plus system, Millipore) with a resistivity of $18.2 \mathrm{M} \Omega \cdot \mathrm{cm}$ was used in all applications.

\subsection{Production and isolation of extracellular ice nucleators}

ECIN's were produced and isolated as described in (Li \& Lee, 1998) . Erwinia herbicola was grown at $18^{\circ} \mathrm{C}$ in a yeast extract. This was followed by the purification of ECINs using centrifugation, sonication, filtration and ultracentrifugation. Then the pellet was suspended in Tris-buffer, freeze-dried and stored at $-20^{\circ} \mathrm{C}$ (Zhu \& Lee, 2007). To evaluate the effect of 
ultracentrifugation speed on ice nucleators, supernatants were centrifuged using a Beckman ultracentrifuge equipped with a 60Ti rotor for 1 hour cumulatively at speeds starting from $10,000 \mathrm{rpm}$ to $50,000 \mathrm{rpm}$. For instance, the $30,000 \mathrm{rpm}$ sample was centrifuged for a total of 3 hours, starting with 1 hour at 10,000 rpm, another hour at 20,000 rpm with the supernatant removed from the first step, and similarly another hour at 30,000 rpm.

\subsection{Nanofilm preparation}

$1 \mathrm{mg} / \mathrm{mL}(0.1 \%)$ solutions of chitosans, carrageenans and pectins were prepared in $0,01 \mathrm{M}$ $\mathrm{NaCl}$ and $0,01 \mathrm{M}$ acetate buffer of $\mathrm{pH}$ 3.74. Carrageenans and pectins were heated at $80^{\circ} \mathrm{C}$ and stirred at this temperature using an impeller for half an hour. Chitosans were stirred overnight and filtered through a $0,45 \mu \mathrm{m}$ sized filter. Incremental bilayers of (Chitosan/Carrageenan) $)_{\mathrm{n}}$ and (Chitosan/Pectin) $)_{\mathrm{n}}(\mathrm{n}$ from 1-6) were fabricated on silicon wafers, to serve as the foundation for the attachment of ECINs. Different molecular weights (1-330 kDa) of chitosan were used to interact with carrageenans of different charge densities (furcellaran, kappa, iota and lambda, in the respective order of low to high charge density). In the second system, carrageenans were replaced with pectins of different degrees of esterification (D.E.) and amidation (D.A.). Multilayer films were deposited onto silicon wafers to act as the positively charged base for ECIN absorption. Silicon wafers were cleaned earlier in a slightly boiled piranha solution (7:3 mixture of $98 \% \mathrm{H}_{2} \mathrm{SO}_{4}$ and $30 \% \mathrm{H}_{2} \mathrm{O}_{2}$ ) for 30 min, then rinsed with copious amount of Milli-Q water and dried with the nitrogen gas. The cleaned silicon wafers of $1 \mathrm{x} 1 \mathrm{~cm}$ size were immersed consecutively in $1 \mathrm{mg} / \mathrm{mL}$ polymer solutions, and finally in different concentrations of ECIN solutions.

For the fabrication of the first layer, wafers were kept in the chitosan solution for one hour, assuring a complete layer of the positively charged polyelectrolyte. For successive layers of pectin/carrageenan, chitosan and ECIN as the top layer, dipping time was 20 
minutes. Wafers were cleaned with Milli-Q water and flushed with gaseous nitrogen each time before dipping in successive solutions. Wafers to be analyzed for surface morphology were placed in a petri dish and covered with parafilm (punched earlier to allow the removal of excessive moisture) and vacuum dried overnight prior to AFM measurement.

\subsection{Atomic force microscopy (AFM)}

Surface morphology was investigated with the Multimode Nanoscope IIIA ${ }^{\text {TM }}$ AFM (Digital Instruments, Veeco Metrology, Santa Barbara, CA, USA), operated in the tapping mode. Silicon tip with an average drive frequency of $265 \mathrm{kHz}$ was used. 1x1, 2x2 and 5x5 micron images of the layers of interest were collected. Section analysis tool was used for the measurements of feature sizes, as the measurements on the $\mathrm{x}$ and $\mathrm{y}$ axes might be misleading due to tip broadening effects. All AFM measurements were carried out in air.

\subsection{Water contact angles}

A VCA Optima video contact angle system (Advance SurfaceTechnology, Billerica, MA, USA) was used to verify the adsorption of layers in a very practical way. This method can be used to confirm surface modification only if the alternating polymers have different wettabilities. The water contact angles of the more hydrophobic top layers will be higher than the relatively hydrophilic ones. Higher water contact angles indicate higher hydrophobicity, because droplets tend to spread better on hydrophilic surfaces.

\section{Results and discussion}

\subsection{Effect of the number of bilayers formed on surface morphology and wettability}

Chitosan / carrageenan system was the first biopolymer pair studied and water contact angle measurements indicated that they can be used to create uniform food grade multilayers. 
This conclusion is supported by the steady variation in contact angles as layers are built on top of each other; indicating improved surface coverage (Figure 1).

Standard deviation of the 10-15 contact angles measured on each layer was within the range of 1.18-3.12, indicating even and homogeneous surfaces. Chitosan of $330 \mathrm{kDa}$ molecular weight was more hydrophobic than iota-carrageenan, as evidenced by the 10 to 15 degrees of difference in the water contact angles as each alternating layer has formed. There is a trend towards increasing contact angles for both polymers with layer build-up, which can be explained by the formation of a thicker multilayer system of higher nanoscale roughness. This is imparted to the surface by the carrageenans that form three dimensional entangled gel networks and random coils, which resemble layers of ropes on top of each other with electrostatically adhesive chitosan layers in between.

Our previous work demonstrated that building more bilayers had an effect on both on the surface morphology and the absorption of ECINs as top layer on the PDDA/PSS system (Gezgin et al., 2013). Here the observations were similar with the AFM images showing more concentrated carrageenan gel networks with thicker and more elevated threads on the surface of higher contrast as the number of bilayers increase (Figure 2). The double helical structure formation proposed in (Bemiller \& Whistler, 1985) as a gelation model for iota carrageenan, is confirmed by the $10^{\text {th }}$ bilayer images that clearly demonstrate those three dimensional networks.

\subsection{Effect of carrageenan type (charge density) on surface morphology and wettability}

Effect of charge density was studied with $10 \mathrm{kDa}$ chitosan as the positively charged polymer to build four different multilayer systems using lambda, iota, kappa carrageenans and furcellaran. 
Looking at the surface morphology of carrageenans on the $6^{\text {th }}$ bilayer, iota carrageenan is different from the other three biopolymers; it forms a uniquely more closely packed and entangled gel network with threads of uniform thickness (Figure 3). In an earlier study, iotacarrageenan was also found to be more suitable for complex formation with oligochitosan leading to more stable capsules (Bartkowiak \& Hunkeler, 2001). Kappa carrageenan, which has a slightly lower charge density than iota, also forms a good gel network. Both kappa and iota carrageenan led to the formation of a mixture of coiled and double-helical structures as seen in the AFM images, which are not seen in the images of lambda carrageenan and furcellaran.

These observations were confirmed by the water contact angle measurements which resulted in higher contact angles for the iota and kappa carrageenan surfaces than the lambda carrageenan and furcellaran surfaces (Figure 4). The formation of the entangled gel network leads to a surface which has higher nanoscale roughness. These entangled gels can hold the pure water droplets together on the surface, not letting them spread, making the contact angles of the water droplets higher. Indeed, the root mean square roughness (RMS) calculated from the AFM images was $1.94 \pm 0.10,2.97 \pm 0.32,3.59 \pm 0.06$ and $1.33 \pm 0.14 \mathrm{~nm}$ for lambda, iota, kappa carrageenans and furcellaran respectively. This data is almost parallel to the water contact angle data where the values are lower for lambda carrageenan and furcellaran than iota and kappa carrageenans, which confirms the proposed correlation between nanoscale surface roughness and surface hydrophobicity.

Except for this instance, interpretation of the water contact angle measurements throughout this study are based on the variation between the wettabilities of the alternating polymer layers making up the system. For example, chitosan layers are characteristically more hydrophobic than carrageenan layers which are relatively hydrophilic, hence water contact angles of the chitosan layers are higher than that of carrageenans (Figure 1). For this 
certain instance where the hydrophilic top carrageenan layers are compared however, water contact angles correlate well with the nanoscale surface roughness as measured by the AFM.

AFM images and water contact angles indicate that the charge densities of different carrageenans are not in a direct correlation with the surface morphology and wettability. Similar properties have been observed with the lambda carrageenan, which has the highest charge density and furcellaran which has the lowest. Appearance of good entangled networks on the nano-thin carrageenan layers which also leads to higher surface hydrophobicity, can be attributed to the special gelling mechanism of kappa and iota carrageenans. How the sulfate groups are positioned on the disaccharide units of each carrageenan is another factor which is critical to the gelling mechanism (Bemiller \& Whistler, 1985). Our data shows that it is critical to the nano-thin layer formation and nanoscale gelation as well. Although lambda carrageenan has the highest charge density among the four and this would ideally lead to better electrostatic interactions with the chitosan layers, it cannot form the three dimensional polymer network that geometrically helps create an ideal nano-thin multilayer structure.

This is due to the presence of two negatively charged sulfate groups positioned on the opposite sides of the saccharide unit, which tend to repel each other. As a result, the polymer chain cannot bend and twist to form the three dimensional double helical structures that the iota and kappa carrageenans form. This makes lambda carrageenan with the highest change density as unsuitable for nano-thin multilayer build up as the furcellaran with the lowest charge density.

\subsection{Effect of chitosan molecular weight on surface morphology and wettability}

Molecular weight effect of chitosan was varied in the range of $1 \mathrm{kDa}$ to $330 \mathrm{kDa}$ to investigate its effect on multilayer build up. Iota-carrageenan which has been observed to be 
the most suitable for multilayer fabrication in the earlier experiments on charge density was used as the negatively charged polyelectrolyte in all systems.

Figure 5 shows the morphological changes when the molecular weight of chitosan is varied in a multilayer system with iota carrageenan. Images of chitosan top layers are almost identical to the carrageenan top layers on the $1 \mathrm{kDa}$ and $10 \mathrm{kDa}$ systems. Only carrageenan gels are visible and not the chitosan molecules which are globular features as observed earlier in the AFM images and also reported in (Haas, Kumar, Borchard, Bakowsky \& Lehr, 2005).

The absence of globular chitosan molecules on these images can be explained by the molecular weight being too small to form visible globules and/or the screening effect of carrageenan gel networks. These AFM images have a height ( $\mathrm{Z}$ scale) of $50 \mathrm{~nm}$, and earlier studies show that the chitosan films are about $20 \mathrm{~nm}$ thick (Assis, Bernardes-Filho, Vieira \& Filho, 2002). Thus the image contrast of $50 \mathrm{~nm}$ used in a chitosan/carrageenan multilayer system is not small enough to see the chitosans among the taller carrageenan gels. When higher molecular weight chitosans are used to build the multilayers however, chitosan layers envelop the carrageenan gels and chitosan globules become more visible. Increased molecular weight also has a tightening effect on the carrageenan gel chains, giving a more closely packed, dense network. This phenomenon has also been observed with the other multilayer systems which will be discussed in the following sections, and advantages of a more concentrated layer of polyelectrolytes will also be addressed.

Figure 6 shows the water contact angles of the chitosan layers seen in Figure 5. There is a positive correlation between the chitosan molecular weight and the hydrophobicity of the layer. Variation of water contact angles during layer build-up is also studied and surface wettability is found to vary more steadily when higher molecular weight chitosans are used (Figure 7). 
This suggests a better surface coverage (i.e. more chitosans on the surface than carrageenans), which also explains the linear increase in water contact angles with increased chitosan molecular weight in Figure 6. By covering the surface more completely, higher molecular weight chitosans lessen the interpenetration of the more hydrophilic layer of carrageenan and makes the chitosan layers more hydrophobic. Thus a broader variation is observed in water contact angles each time a new layer is added.

3.4. Effect of chitosan molecular weight on surface morphology and wettability in the chitosan/pectin/ECIN multilayer system

As with the carrageenan experiments, chitosan molecular weight was varied first and investigated by water contact angle measurements and AFM imaging. Danisco Grindsted LA 410 pectin, with an esterification degree of $29-33 \%$ and amidation degree of $20 \%$ was used to build the three multilayer systems. LA410 pectin was significantly more hydrophilic than the other biopolymers (Figure 8). As seen earlier with the carrageenan system, chitosan and ECIN shows a trend towards increasing hydrophobicity when higher molecular weight chitosans are used, confirming better surface coverage.

Increasing water contact angles of the ECIN layers on top of higher molecular weight chitosans indicate better attachment of ECINs to the surface. However, this can also be attributed to the increased nanoscale roughness of the higher molecular weight chitosan layers. As discussed earlier when comparing the wettability of different types of carrageenans, nanoscale surface roughness contributes to hydrophobicity by preventing water droplets from spreading wider on the surface. Ability of higher molecular weight chitosan to form a more closely packed pectin gel network also makes a contribution, since the number of carboxyl groups per unit area increases and with the uniform charge distribution more homogeneous layers are achieved. 
The multilayer systems created with the $130 \mathrm{kDa}$ and $330 \mathrm{kDa}$ chitosans were tested for ice nucleation activity on the cooling bath. Silicon wafers which had either chitosan or pectin on top of the $6^{\text {th }}$ bilayer, remained unfrozen for over an hour at $-8^{\circ} \mathrm{C}$, where nucleation occurred within five minutes with the wafers which had ECIN on top of the (Chitosan/Pectin) $)_{5}$ Chitosan multilayer system.

AFM images of top chitosan, pectin and ECIN layers were obtained for all three systems (Figure 9). As with the carrageenan/chitosan multilayers, chitosan top layers did not look significantly different from top pectin layers. On the ECIN top layer images, the globular features are more clearly visible than they are on the iota-carrageenan system, because the gel networks of pectin appear as thinner threads than iota-carrageenan threads on which the ECIN globules are more identifiable.

The shapes of branched pectin chains alone and in interaction with proteins were very similar to how they looked in the AFM images of a previous study (Kirby, MacDougall \& Morris, 2008). Using high resolution AFM images (scanned $1 \mathrm{x} 1 \mu \mathrm{m}$ and further magnified allowing the use of a $100 \mathrm{~nm}$ scale bar), they were able to show how pectin threads interact with the protein globules either by surrounding them or getting attached to them through the edges of the chain (Kirby et al., 2008), again via the electrostatic interactions of the oppositely charged groups they possess.

3.5. Effect of pectin degree of esterification on surface morphology and wettability in the chitosan/pectin/ECIN multilayer system

Effect of degree of esterification on wettability and surface morphology during the layer build-up process was investigated using Grindsted LA410 pectin with 29-33\% esterification and 20\% amidation and Sigma P9561 citrus pectin with 90\% esterification. 
AFM images show that multilayers could not be fabricated successfully with the highly esterified Sigma P9561 citrus pectin system thus images are significantly different from those with the LA410 pectin; the surface is relatively smooth with no pectin gel networks, only precipitates and isolated threads (Figure 10). The cooling bath experiments also verified the failure to fabricate a robust multilayer system with a functioning ECIN top layer. The droplets on these wafers did not freeze where those on the low methoxyl pectin systems both with and without amidation got frozen successfully at $-8^{\circ} \mathrm{C}$ in five minutes.

This observation serves as a supplementary evidence to support the hypothesis that the attachment of polymers to each other during multilayer build-up is driven by electrostatic interactions for the most part. Pectins with high degree of esterification have mostly methyl esterified carboxylic acid groups on the galacturonic acid backbone. These groups are not available to interact electrostatically with the amide groups of chitosan. Low methoxyl pectins however have copious free carboxylic acid groups. In some pectins these free groups can be amidated, effect of which was also investigated and discussed in the following section.

3.6. Effect of pectin degree of amidation on surface morphology and wettability in the chitosan/pectin/ECIN multilayer system

Effect of amidation degree was studied using $130 \mathrm{kDa}$ chitosan and two pectins with similar degrees of esterification: One of them, LC 410 (DE: 29-33\%, DA:20\%) is amidated; and the other, LA950 (DE: 31-33\%, DA:0) is not. Surface morphologies of the two systems were not as significantly different as what was observed with the high methoxyl vs. low methoxyl systems (Figure 10). Pectin and chitosan top layers look almost identical except for the pectin gels are more clearly visible in the amidated system. However looking at the ECIN images, globular features were more frequent than what was observed on the system with the non-amidated pectin (Figure 11). 
To test this observation, water contact angles of these surfaces were collected. Contact angles of the similar looking chitosan top layers were very close to each other. Pectin top layer of the amidated system showing more of the clearly visible gels in the AFM images had lower contact angles than the non-amidated system (Figure 12). This suggests a better surface coverage on the amidated system, since earlier contact angle measurements where chitosan molecular weight was varied showed that pectin layers are significantly more hydrophilic than the ECIN and chitosan layers (Figure 8). Thus the characteristic of higher wettability is more apparent with the amidated system. Similarly, the ECIN top layer of the amidated system has higher contact angles than the ECIN on the non-amidated system. It also had higher contact angles in Figure 8 against pectin and chitosans of different molecular weights. This serves well as an indicator of better ECIN adsorption, and correlates well with the AFM images showing a denser layer of ECIN globules.

Although amidated carboxyl groups are no longer available as negatively charged free groups, amidation can make a contribution to the multilayer system by allowing stronger electrostatic interactions between the pectin layers that sandwich the chitosan layers. It should be kept in mind that those layers do not have perfect surface coverage over the underlying layers. So there are always spots or regions of the lower layers.. Inevitable interpenetration of these layers highlights the importance of amidated groups on the pectin galacturonic acid backbone. They allow a stronger interaction with both the chitosan layer and also with the pectin layer under the chitosan where negative charges are dominant, holding both layers together more robustly. This can also be explained by the presence of a threshold for the required degree of esterification. Having less than $10 \%$ of free carboxyl groups is not enough to build multilayers, where with $50 \%$ and $70 \%$ there is no big difference, because in both systems there are enough groups to allow electrostatic interactions between the polyelectrolytes. 


\section{Conclusion}

This research hypothesized that synthetic polyelectrolytes can be replaced by biopolymers to build functional, food grade multilayer systems via the LbL deposition method. Our findings demonstrate successfully engineered nano-thin multilayers using food grade alternatives. The functionality of the food grade multilayers have been tested with the ice nucleation application, which serves a potential to provide significant energy savings in the freezing and cold storage of products. Iota-carrageenan performs better than others due to the high charge density and ideal positioning of charged groups. Higher MW chitosans provide better surface coverage and lead to the formation of more closely packed gel networks when paired with carrageenans and pectins. Amidated low methoxyl $(<50 \% \mathrm{DE})$ pectins form optimum multilayer systems for ECIN absorption. Availability of enough free carboxylic acid groups is the main factor when choosing the right pectin (90\% DE was too high), where amidation had a secondary effect on building up a better multilayer foundation for ECIN absorption. Overall, we recommend iota carrageenan and amidated low methoxyl pectins as the negatively charged and high molecular weight chitosan (130 and $330 \mathrm{kDa}$ both perform well) as the positively charged biopolymers to fabricate a robust food grade multilayer system. Poly epsilon-lysine ( $\varepsilon$-PL) will be studied as an alternative to chitosan in the next step, which has several advantages such as better solubility in water and GRAS status since July 2003 in the range of 5-50 ppm (chitosan is "self-affirmed" GRAS). Further investigation on alternative polyelectrolyte multilayer systems can be helpful for specific applications, where there may be certain limitations for the choice of ingredients (ingredient compatibility, sensory concerns, regulatory limitations, etc.). Experimentation with other food polymers such as starches, gelatin, gum arabic and alginic acid can widen the range of 
possible food applications. AFM images can be supplemented by cross-section SEM images to confirm the structural changes occurring through the nanoscale layer build up process.

\section{Acknowledgements}

This study was supported in part by USDA-NRI, Center of Advanced Food Technology (CAFT) and TÜBİTAK (The Scientific and Technological Research Council of Turkey). 
Assis, O. B. G., Bernardes-Filho, R., Vieira, D. C., \& Filho, S. P. C. (2002). AFM Characterization of Chitosan Self-Assembled Films. International Journal of Polymeric Materials, 51(7), 633 - 638.

Bartkowiak, A., \& Hunkeler, D. (2001). Carrageenan-oligochitosan microcapsules: optimization of the formation process. Colloids and Surfaces B: Biointerfaces, 21(4), 285.

Bemiller, J. N., \& Whistler, R. L. (1985). Carbohydrates. In O. R. Fennema (Ed.), Food Chemistry (3rd ed., pp. 158-221). New York and Basel: Marcel Dekker, Inc.

Cai, K., Rechtenbach, A., Hao, J., Bossert, J., \& Jandt, K. D. (2005). Polysaccharide-protein surface modification of titanium via a layer-by-layer technique: Characterization and cell behaviour aspects. In Biomaterials (Vol. 26, pp. 5960).

Decher, G. (1997). Fuzzy Nanoassemblies: Toward Layered Polymeric Multicomposites. Science, 277(5330), 1232-1237.

Duman, J. G. (2001). Antifreeze and Ice Nucleator Proteins in Terrestrial Anthropods. Annu. Rev. Physiol., 63, 327-357.

Gezgin, Z., Lee, T. C., \& Huang, Q. (2013). Engineering functional nanothin multilayers on food packaging: ice-nucleating polyethylene films. J Agric Food Chem, 61(21), 51305138.

Haas, J., Kumar, M. N. V. R., Borchard, G., Bakowsky, U., \& Lehr, C.-M. (2005).

Preparation and Characterization of Chitosan and Trimethyl-chitosan-modified Poly(E-caprolactone) Nanoparticles as DNA Carriers. AAPS PharmSciTech., 6(1), 22-30.

Hugerth, A., \& Sundelöf, L.-O. (2001). The effect of polyelectrolyte counterion specificity, charge density, and conformation on polyelectrolyte-amphiphile interaction: The carrageenan/furcellaran-amitriptyline system. Biopolymers, 58(2), 186-194.

Kirby, A. R., MacDougall, A. J., \& Morris, V. J. (2008). Atomic force microscopy of tomato and sugar beet pectin molecules. Carbohydrate Polymers, 71(4), 640.

Kujawa, P., Moraille, P., Sanchez, J., Badia, A., \& Winnik, F. M. (2005). Effect of Molecular Weight on the Exponential Growth and Morphology of Hyaluronan/Chitosan Multilayers:\&nbsp; A Surface Plasmon Resonance Spectroscopy and Atomic Force Microscopy Investigation. Journal of the American Chemical Society, 127(25), 92249234.

Li-Jung, Y., Chen, M.-L., Tzeng, S.-S., Chiou, T.-K., \& Jiang, S.-T. (2005). Properties of extracellular ice-nucleating substances from Pseudomonas fluorescens MACK-4 and its effect on the freezing of some food materials. Fisheries Science, 71(4), 941-947.

Li, J., \& Lee, T. C. (1998). Bacterial Extracellular Ice Nucleator Effects on Freezing of Foods. Journal of Food Science, 63(3), 375-381.

Shumilina, E. V., \& Shchipunov, Y. A. (2002). Chitosan-Carrageenan Gels. Colloid Journal, 64(3), 372.

Worth, H. G. J. (1967). The Chemistry and Biochemistry of Pectic Substances. Chemical Reviews, 67(4), 465-473.

Zhang, X., Chen, H., \& Zhang, H. (2007). Layer-by-layer assembly: from conventional to unconventional methods. Chemical Communications, 1395-1405.

Zhu, X., \& Lee, T.-C. (2007). Application of a biogenic extra cellular ice nucleator for food processing: effects on the freeze-thaw stability of fish actomyosin from tilapia.

International Journal of Food Science \& Technology, 42(6), 768-772. 


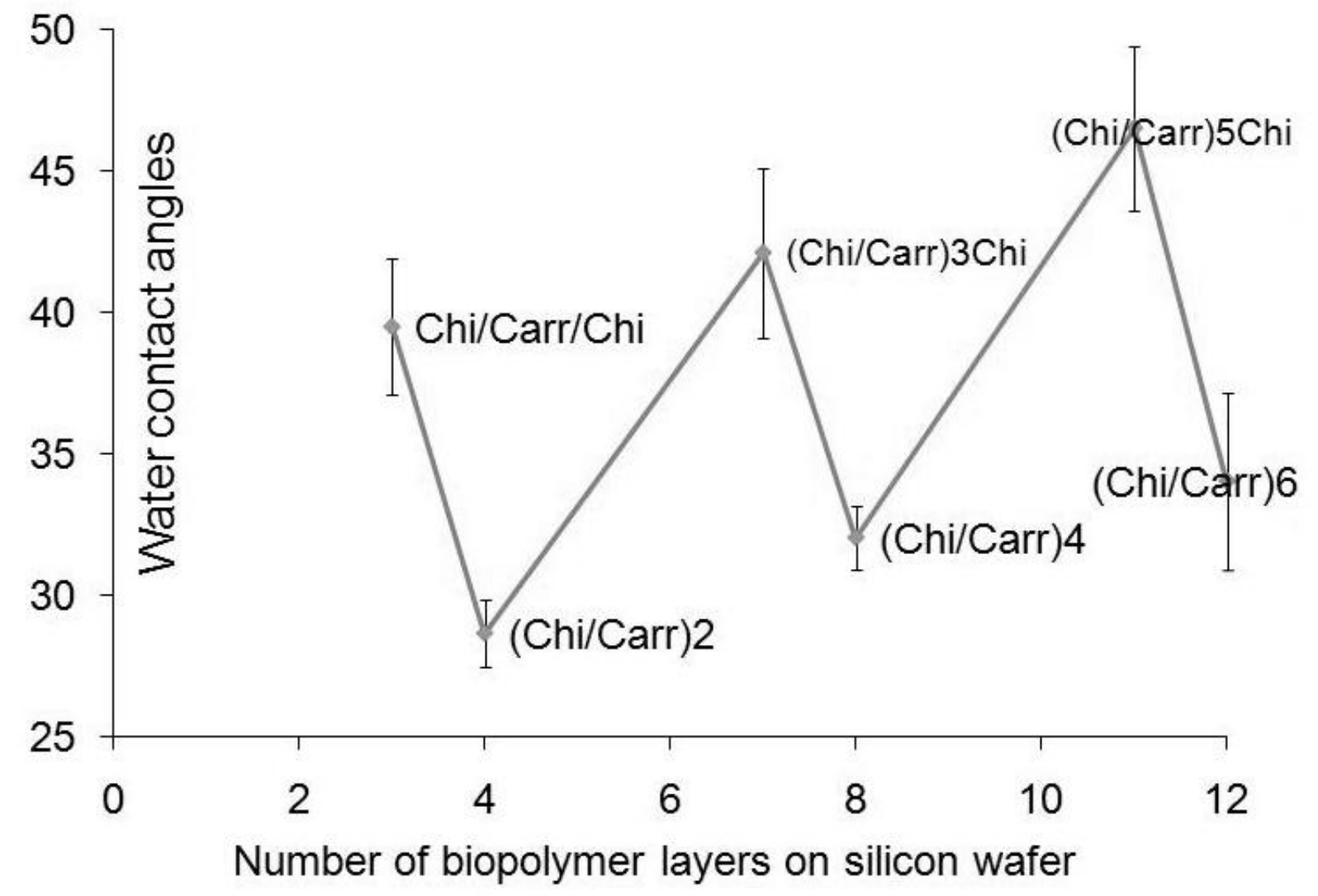

Figure 1. Variation in water contact angles as multilayers are fabricated using chitosan of $330 \mathrm{kDa}$ molecular weight and iota-carrageenan. 


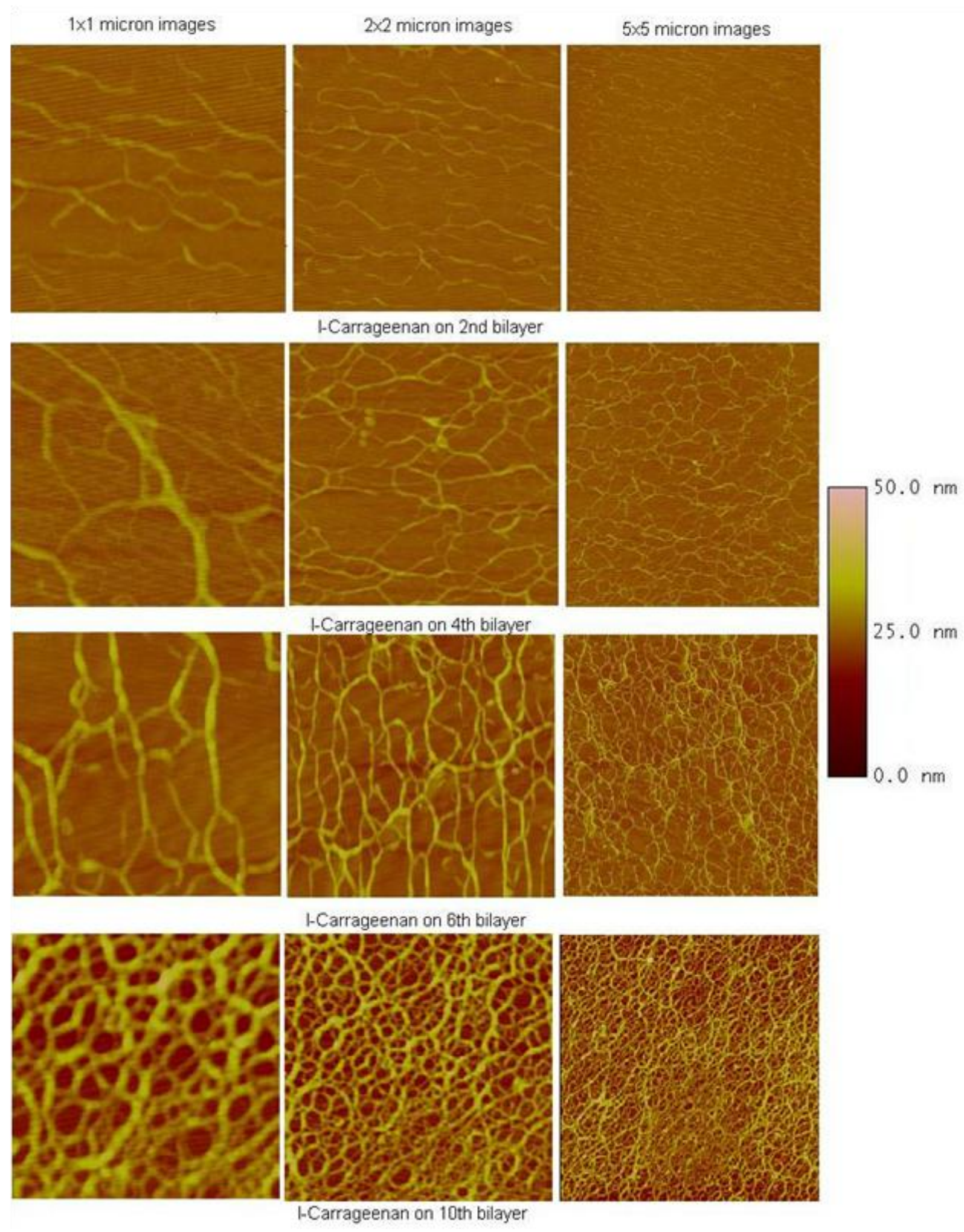

Figure 2. AFM height images of top iota-carrageenan layers, as the top layer of 2 to 10 bilayers of $10 \mathrm{kDa}$ chitosan and iota-carrageenan. 

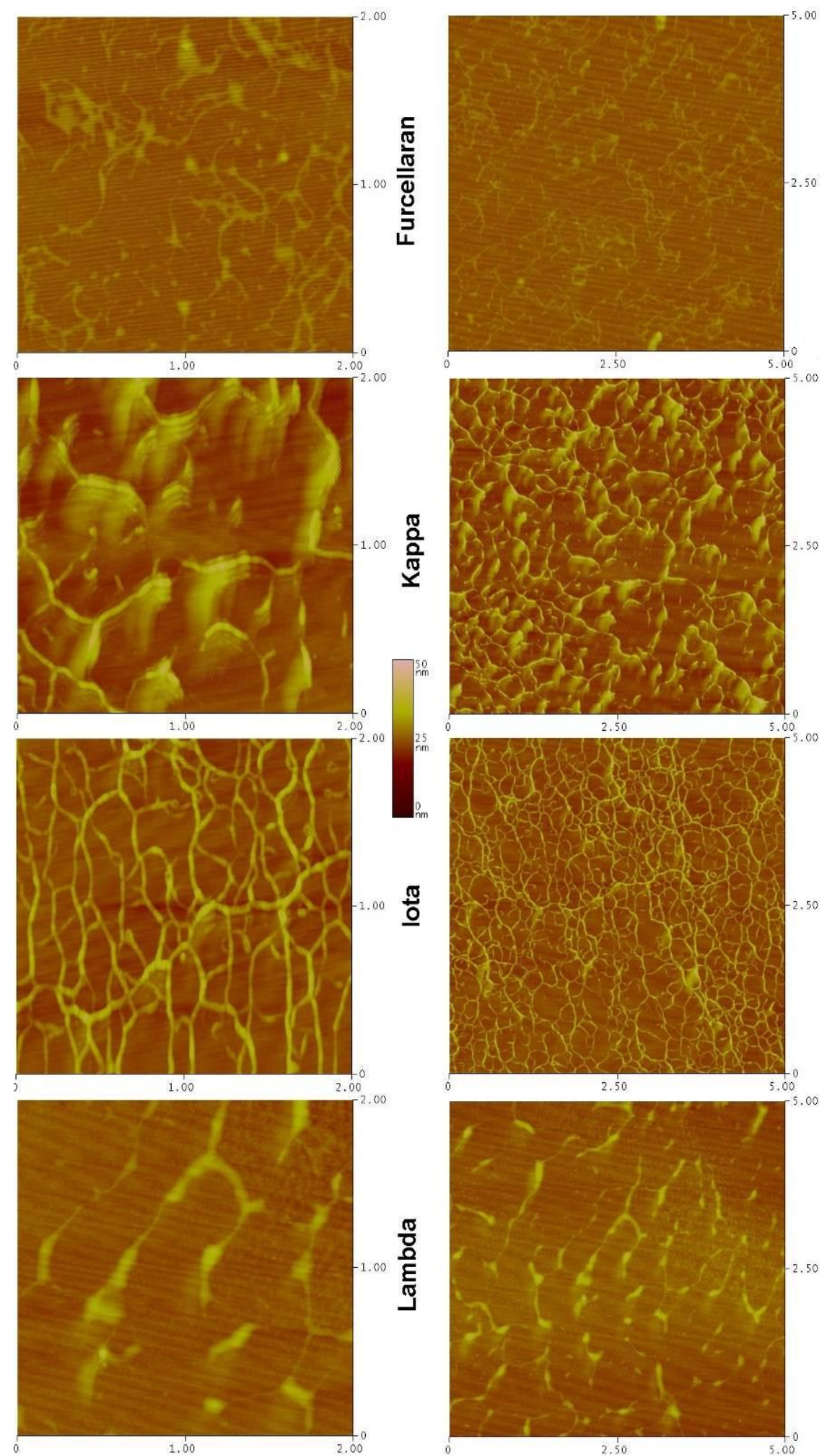

Figure 3. $2 \times 2$ and $5 \times 5 \mu \mathrm{m}$ AFM images of top carrageenan and furcellaran layers on the 6th bilayer, paired with $10 \mathrm{kDa}$ chitosan. 


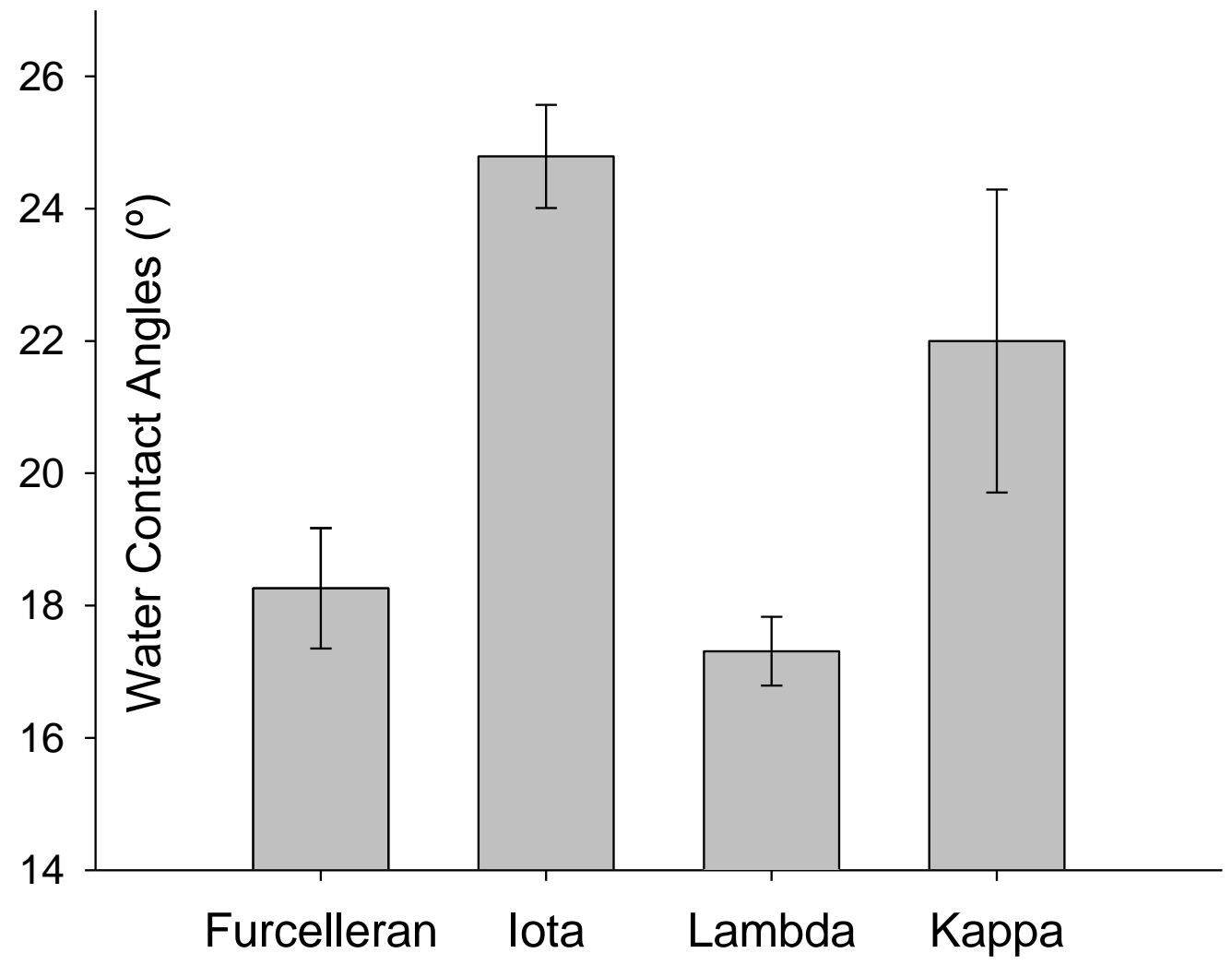

Figure 4. Water contact angles of top carrageenan and furcellaran layers on 6 bilayers with standard deviations. $10 \mathrm{kDa}$ chitosan was used in all multilayers. 


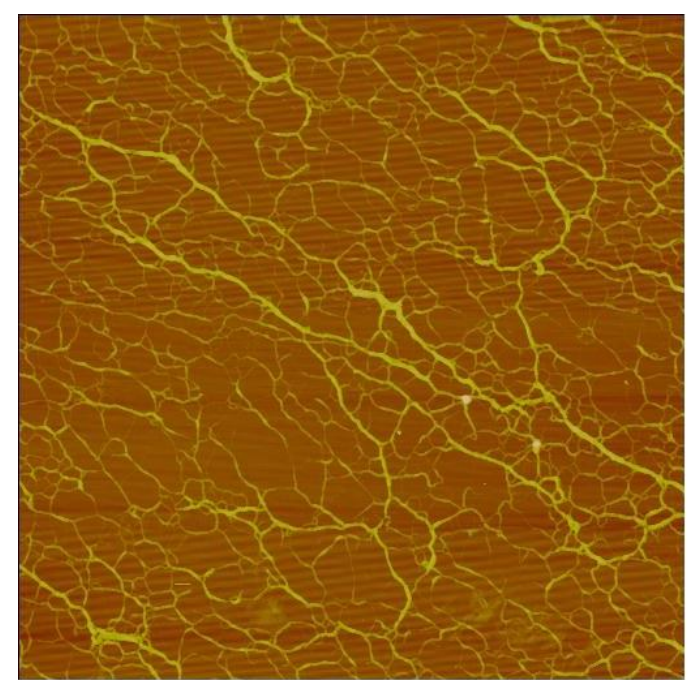

$1 \mathrm{kDa}$

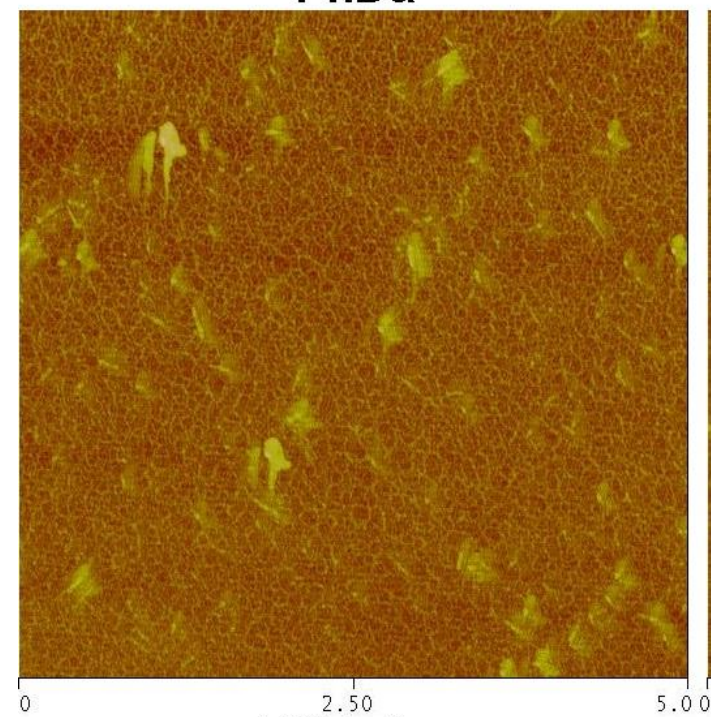

$130 \mathrm{kDa}$

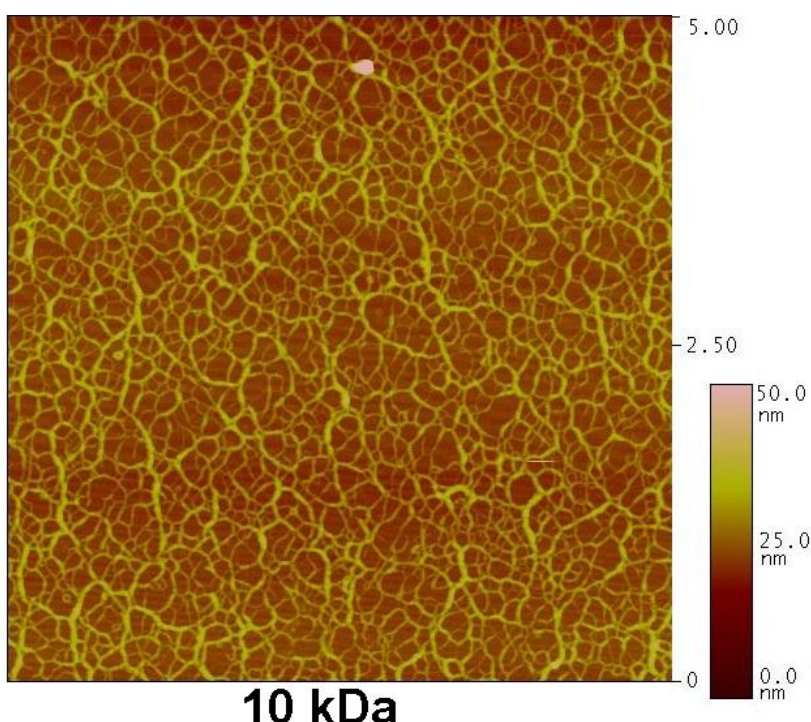

$10 \mathrm{kDa}$

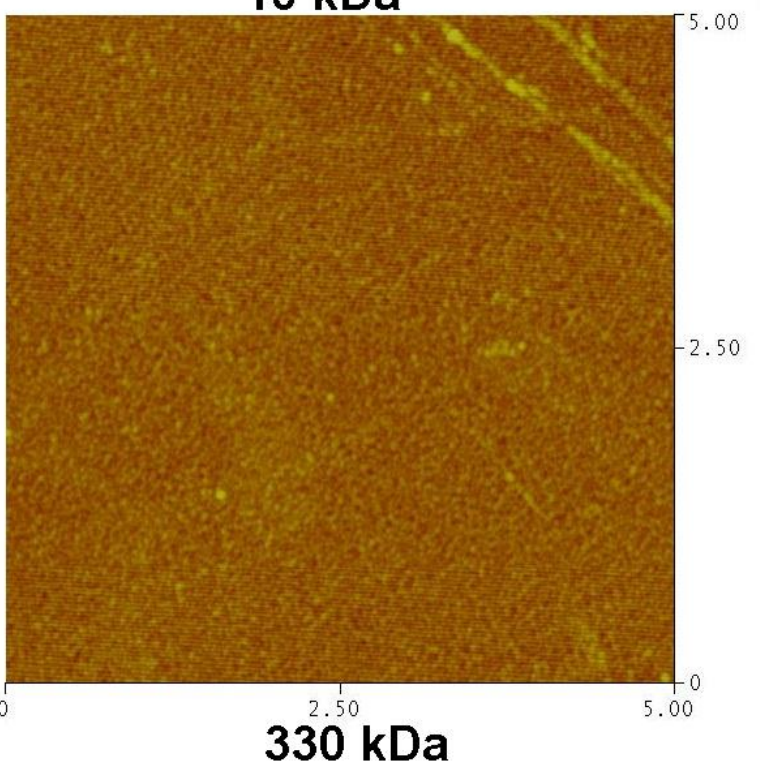

Figure 5. $5 \times 5 \mu \mathrm{m}$ AFM images of top chitosan layers on 5 chitosan/iota carrageenan bilayers where the molecular weight of chitosan was varied between $1 \mathrm{kDa}$ to $330 \mathrm{kDa}$. 


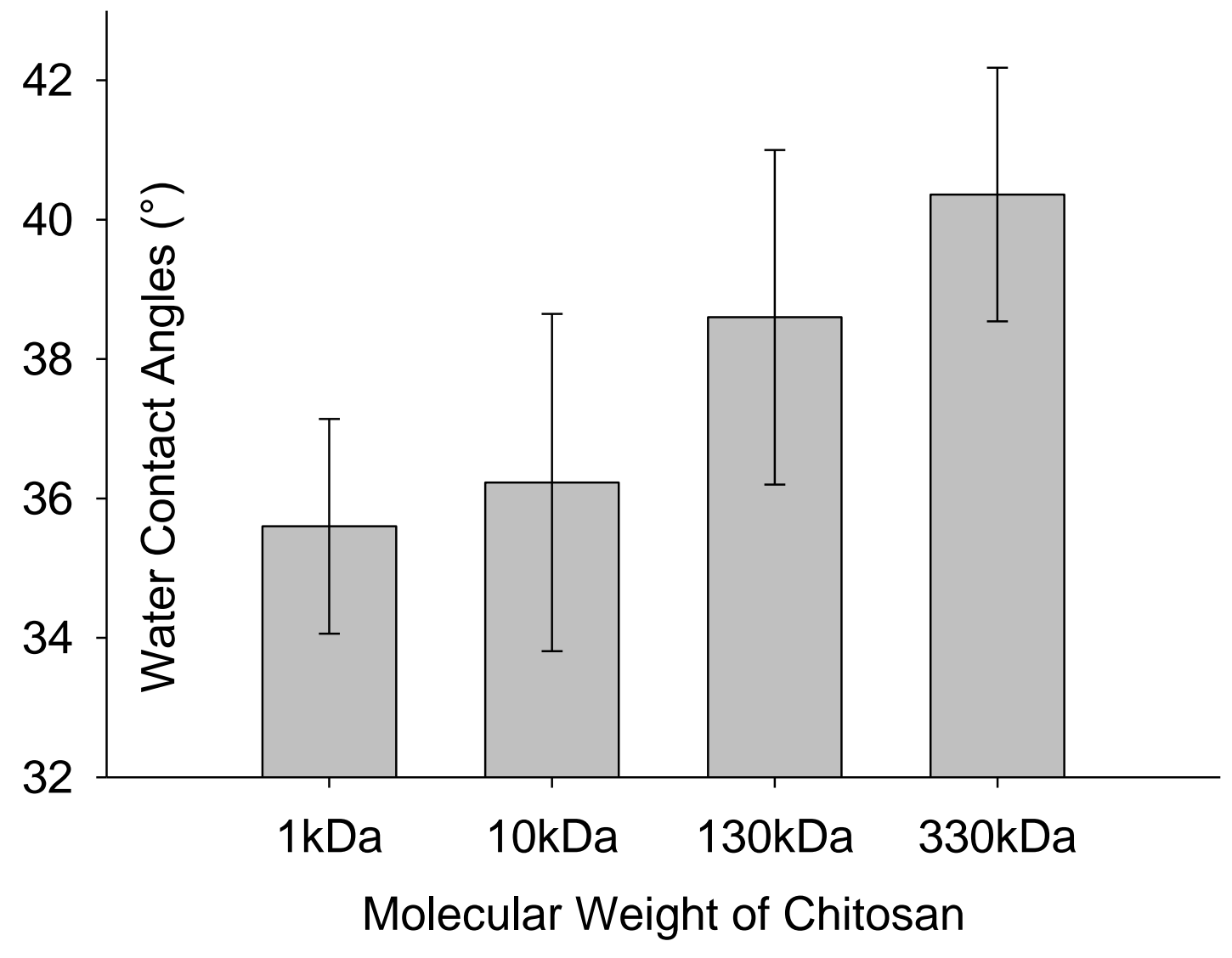

Figure 6. Water contact angles of top chitosan layers on 5 chitosan/iota carrageenan bilayers where the molecular weight of chitosan was varied between $1 \mathrm{kDa}$ to $330 \mathrm{kDa}$. 


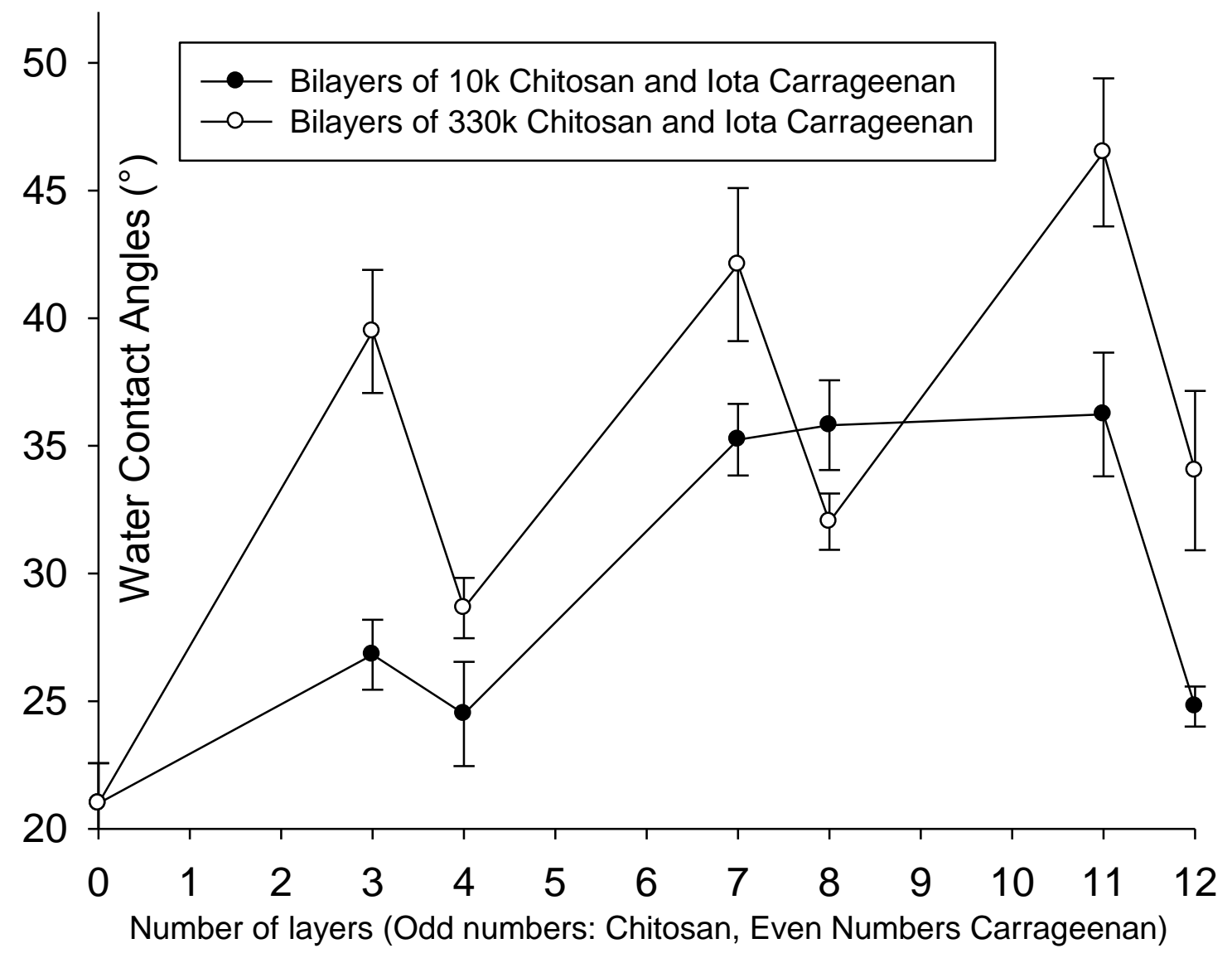

Figure 7. Variation of water contact angles with layer build up for the $10 \mathrm{kDa}$ and $330 \mathrm{kDa}$ chitosan systems with iota carrageenan 


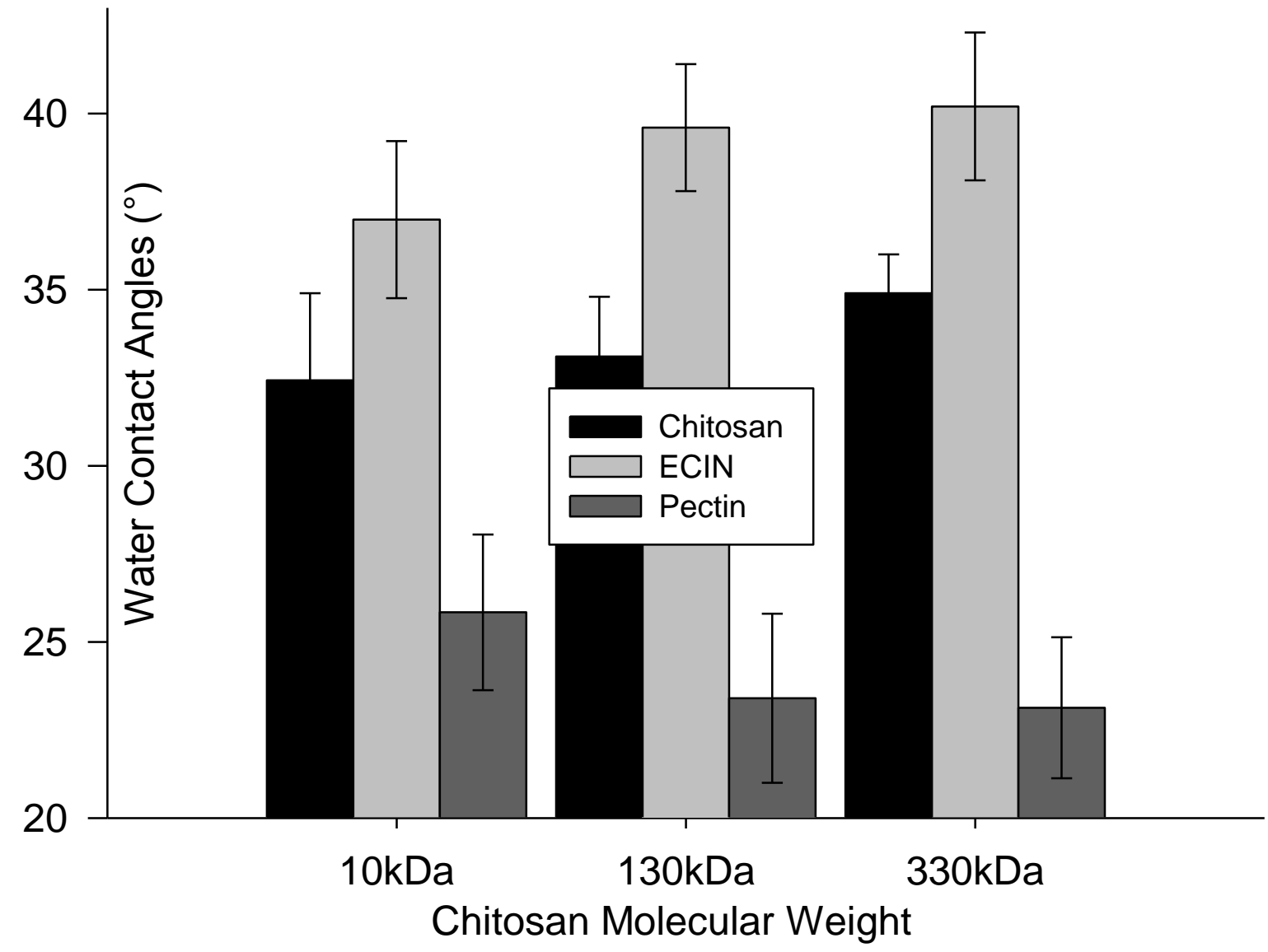

Figure 8. Water contact angles of top chitosan, pectin and ECIN layers on the $6^{\text {th }}$ bilayer when paired with 10, 130 and 330kDa chitosans. 

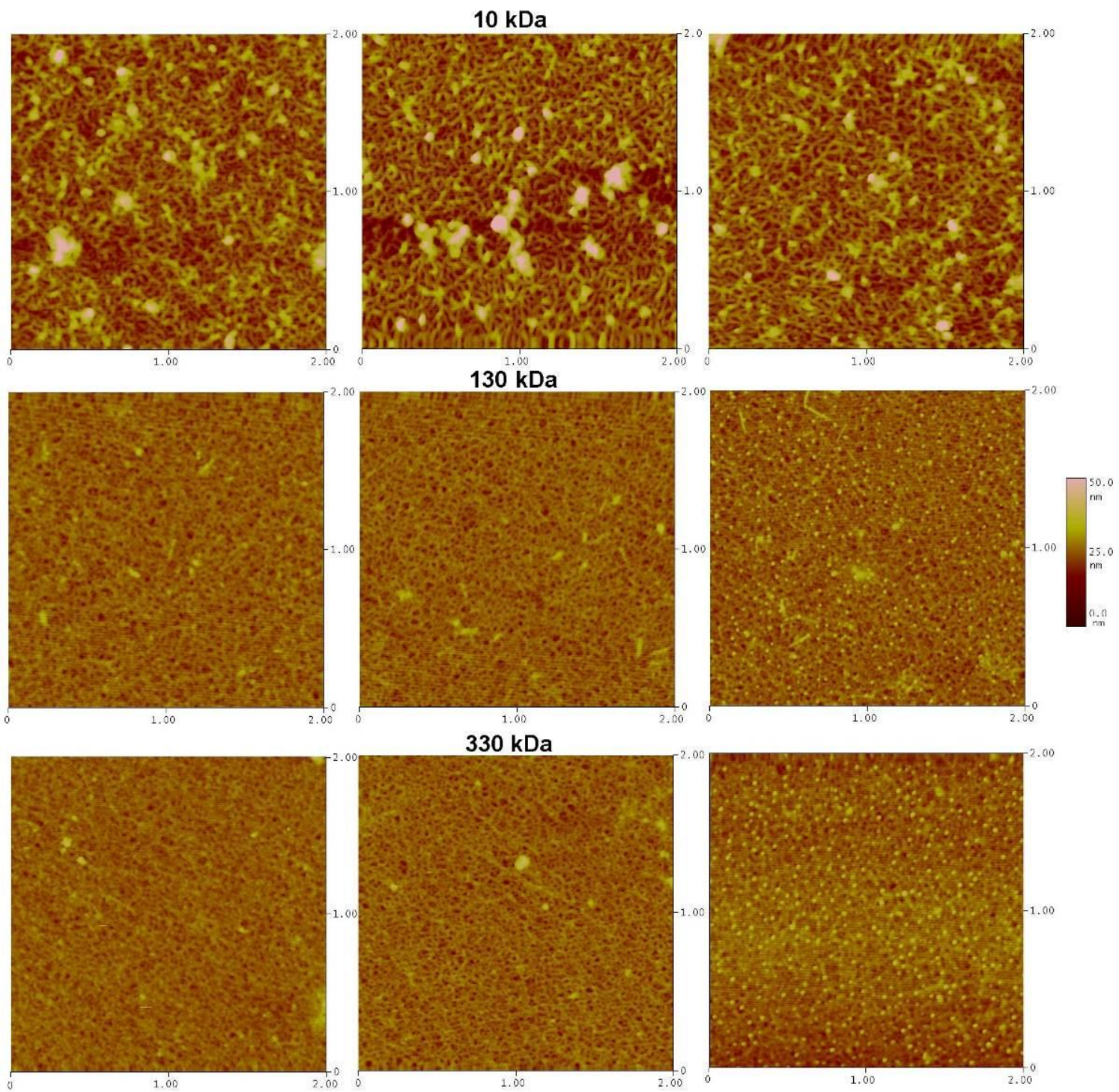

Chitosan

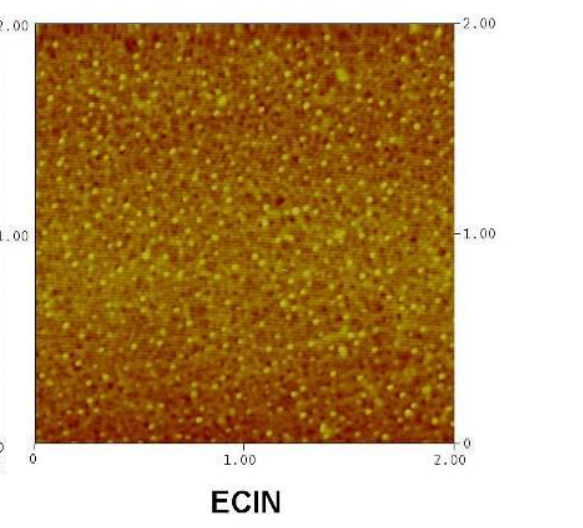

Figure 9. $2 \times 2 \mu \mathrm{m}$ AFM images of top pectin, chitosan and ECIN layers on the $6^{\text {th }}$ bilayer of 10,130 or $330 \mathrm{kDa}$ chitosan / LA410 pectin multilayer systems. 

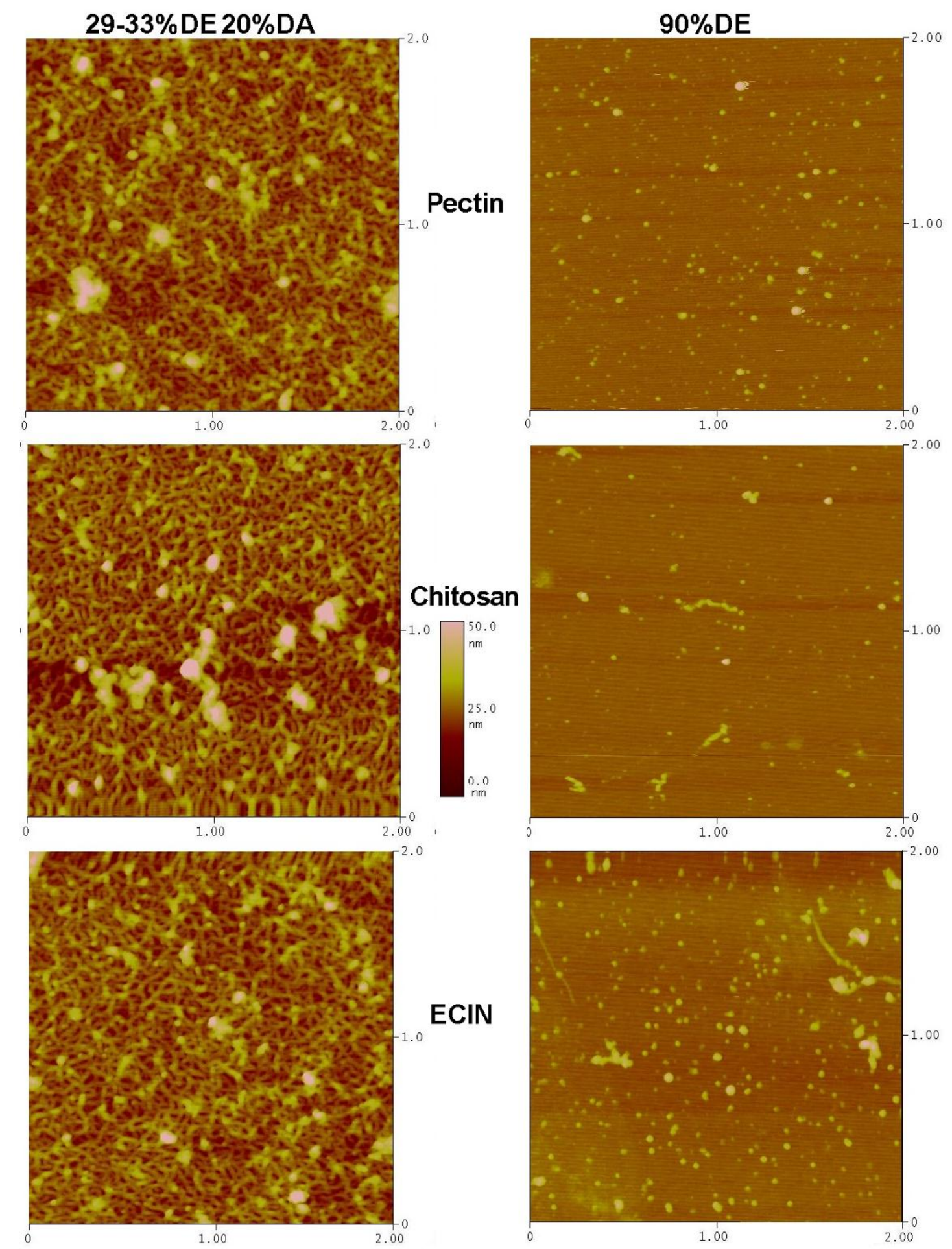

Figure 10. $2 \times 2 \mu \mathrm{m}$ AFM images to show the effect of esterification on layer build-up in the $10 \mathrm{kDa}$ chitosan/LA410 (29-33\%DE with 20\%DA, left row) or P9561 (90\%DE- no amidation,right row) pectin multilayer systems. 
$29-33 \%$ DE $20 \%$ DA
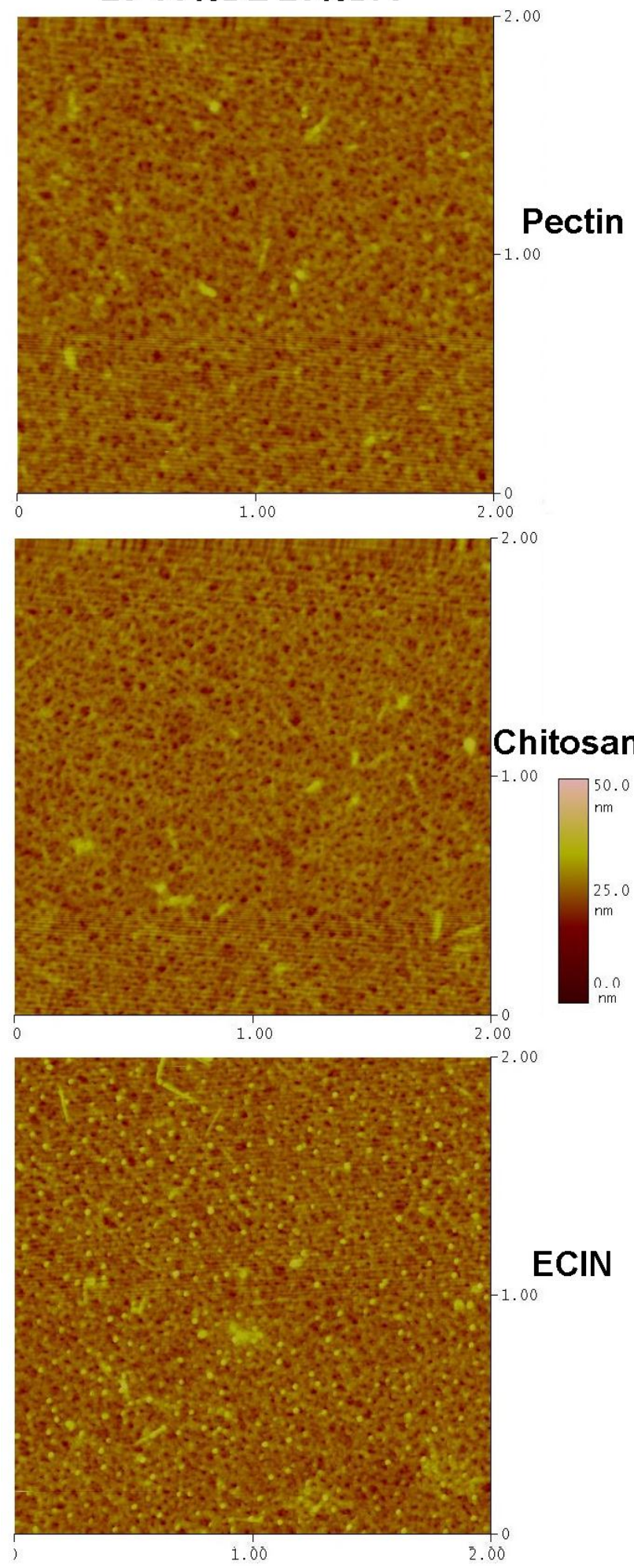

$31-33 \%$ DE
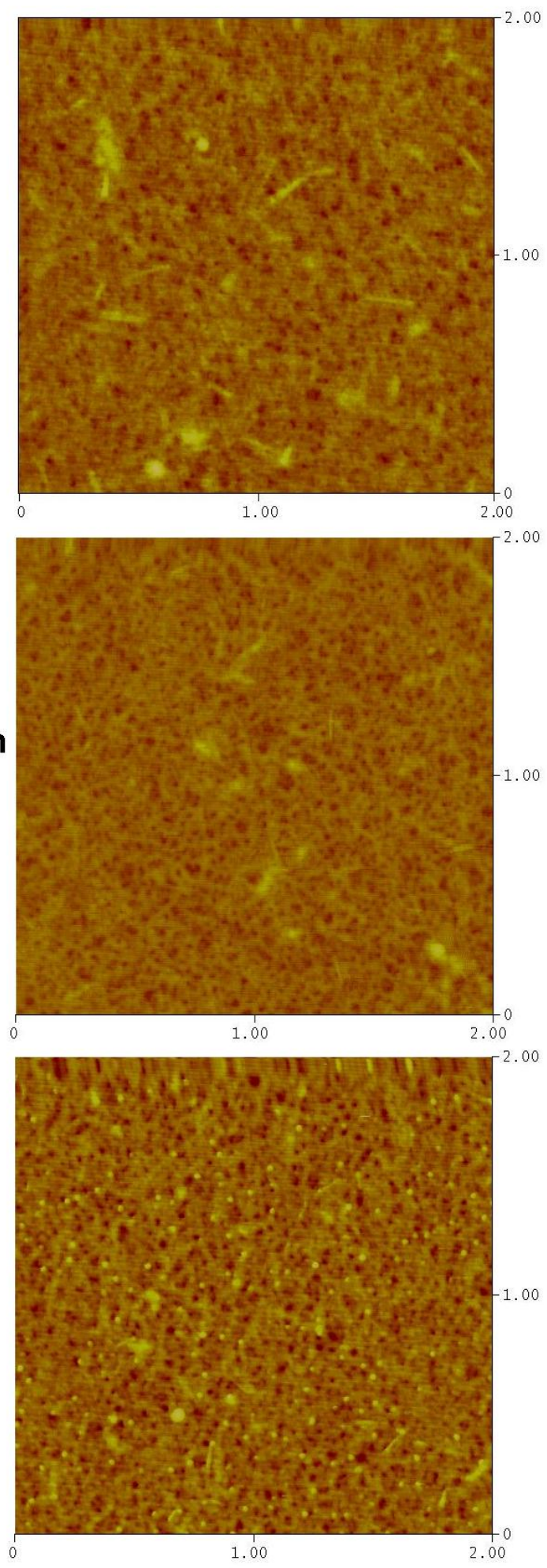

Figure 11. $2 \times 2 \mu \mathrm{m}$ AFM images top Chitosan, ECIN and Pectin layers with water contact angles \pm standard deviation on two multilayers systems with $130 \mathrm{kDa}$ chitosan and either LC950 or LA 410 pectin. 


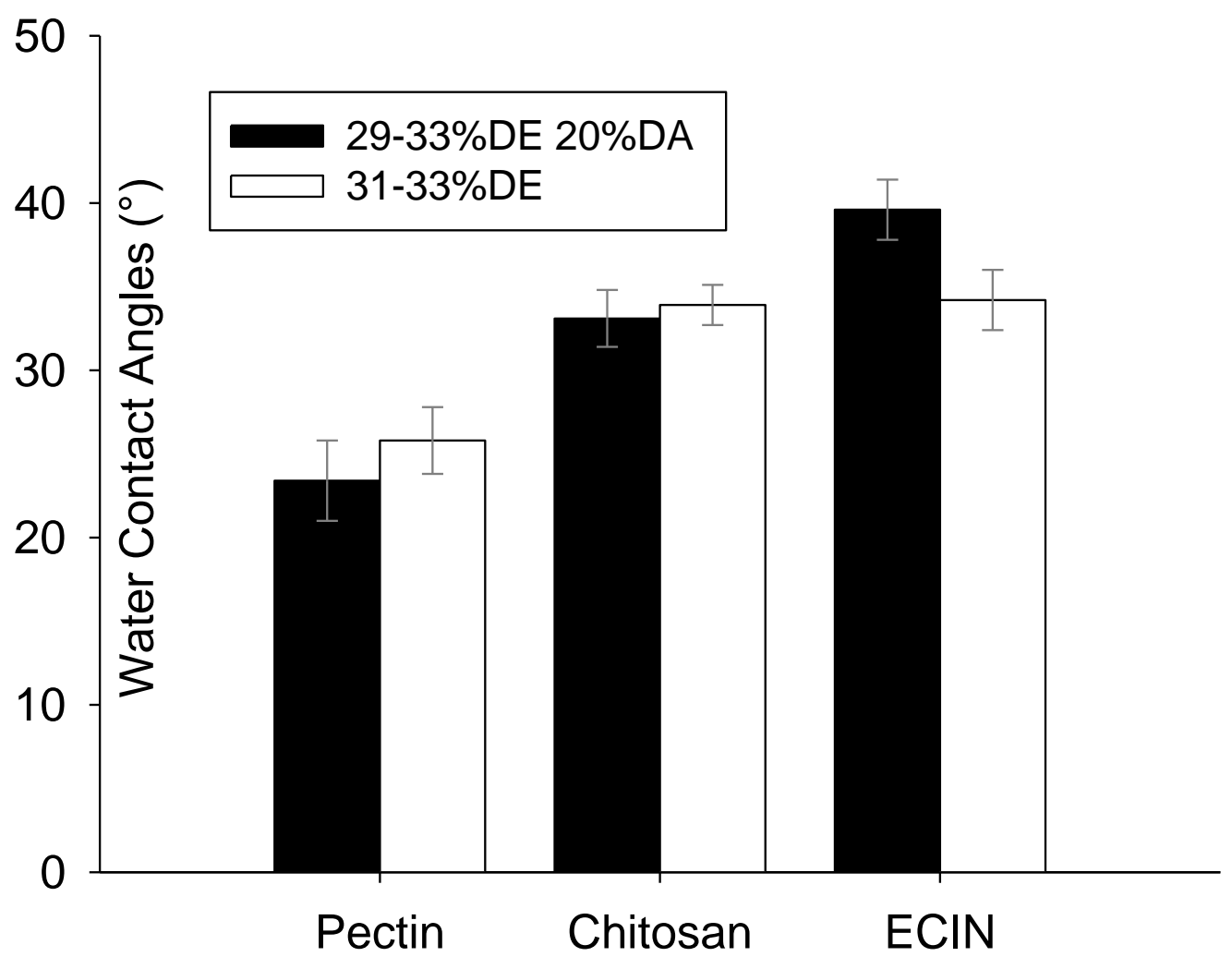

Figure 12. Water contact angles of the top pectin, chitosan and ECIN layers of the $130 \mathrm{kDa}$ Chitosan paired with amidated (20\%) and non-amidated pectin of similar esterification. 